\title{
Hole-Free Phase Plate Energy Filtering Imaging of Graphene: Toward Quantitative Hole-Free Phase Plate Imaging in a TEM
}

\author{
Marek Malac ${ }^{\Delta \circ}$, Emi Kano ${ }^{\Delta \circ}$, Misa Hayashida ${ }^{\Delta}$, Masahiro Kawasaki*, Sohei Motoki*, Ray \\ Egerton $^{\circ}$, Isamu Ishikawa ${ }^{\square}$, Yoshio Okura ${ }^{\square}$, Marco Beleggia \\ ${ }^{\Delta}$ National Institute of Nanotechnology, 11421 Saskatchewan Drive, Edmonton, Canada. \\ - Department of Physics, University of Alberta, T6G 2E1, Edmonton, Canada. \\ * JEOL USA Inc., 11 Dearborn drive, Peabody, MA, USA. \\ JEOL Ltd. 1-2 Musashino 3 chome, Akishima, Tokyo 198-8558, Japan. \\ - Center for Electron Nanoscopy, Technical University of Denmark, 2800 Lyngby, Denmark.
}

Hole free phase plate (HFPP) imaging has been developed to increase TEM phase contrast for qualitative imaging purposes [1] in the sense that the image intensity is not related to sample properties in a simple manner. Here we report simultaneous utilization of HFPP and energy filtered TEM (EFTEM) thickness mapping as a first step toward quantitative HFPP imaging, using a contaminated graphene film as test specimen.

The data were collected in a JEOL JEM 2200 FS TEM operated at $200 \mathrm{kV}$ with a Schottky electron source and an in-column omega filter. The HFPP is placed at the in-gap objective aperture plane, enabling us to utilize microscope magnification between 1500x and $1 \mathrm{Mx}$. The carbon phase plate was heated during operation to about $250{ }^{\circ} \mathrm{C}$ and data were collected after the HFPP was allowed to settle for about $100 \mathrm{~s}$.

Figure 1 shows conventional a) and HFPP b) images of the graphene film at 50kx magnification. The contrast in a) is higher in b). A thickness map obtained from logarithm of the ratio between elastic and inelastic electrons is shown in c) for data obtained without HFPP [2]. A HFPP-EFTEM thickness map obtained by the same log ratio method of zero-loss filtered and unfiltered HFPP images is shown in d). e) shows a HFPP-EFTEM thickness map obtained from the area marked by the red rectangle in d) at $300 \mathrm{kx}$ magnification. The maximum thickness variation in e) is about 0.06 inelastic mean free path $\lambda_{1}$. A EFTEM thickness map without HFPP over the same are as in e) did not yield interpretable data and was difficult to align due to presence of fringes arising from spherical aberration. Profiles along the lines indicated in c) and d), are shown in f). The upper profile in e) corresponds to the HFPP thickness map (red, dashed) from d) and the lower (blue, solid) profile to a thickness map without HFPP, derived from c). The HFPP-EFTEM thickness map appears to provide more spatial resolution that the standard EFTEM thickness map and thinner contamination islands can be observed in the HFPP-EFTEM map more clearly than in the standard EFTEM map. The profiles in $\mathrm{f}$ ) indicate that the addition of the HFPP in the beam for the log-ratio EFTEM thickness mapping results in addition of $\sim 0.04$ inelastic mean free paths $\lambda$ across the field of view. The features in the HFPP-EFTEM thickness map appears to be more localized than in EFTEM thickness map without HFPP. Taking into account the known thickness of the carbon HFPP of $\sim 13 \mathrm{~nm}$, the $0.04 \lambda_{2}$ implies $\sim 0.4 \mathrm{mrad}$ collection angle for an object placed in the objective aperture plane rather than in sample plane.

Assuming an inelastic mean free path $\lambda_{1}=114 \mathrm{~nm}$ for a $200 \mathrm{keV}$ beam in carbon at the sample plane (100 mrad collection angle adequate for no objective aperture), the estimated thickness in the sample region is $17.6 \pm 0.5 \mathrm{~nm}$ in HFPP-EFTEM (marked with yellow band in $\mathrm{f}$ ) and $13.5 \pm 0.5 \mathrm{~nm}$ from the standard EFTEM thickness map. 
We have also been able to utilize HFPP-EFTEM imaging for plasmon-energy observation of Ge layers in Si single crystal MAGICAL ${ }^{\mathrm{TM}}$ sample (not shown) suggesting that HFPP-EFTEM may be suitable for chemical mapping in a TEM. The log-ratio EFTEM thickness mapping method appears to yield higher spatial and thickness resolution than the standard EFTEM log-ratio thickness mapping without HFPP in the electron beam.

[1] M. Malac et. al., Ultramicroscopy 118 (2012), p. 77.

[2] R. F. Egerton, EELS in the TEM $2^{\text {nd }}$ ed., Springer Verlag, 2011.

[3] Support of NINT and JEOL USA Inc. is acknowledged. The work was made possible by close collaboration with team at JEOL Ltd. Dr. Y. Okura, Dr. I. Ishikawa, Y. Konyuba and Dr. Hosogi.
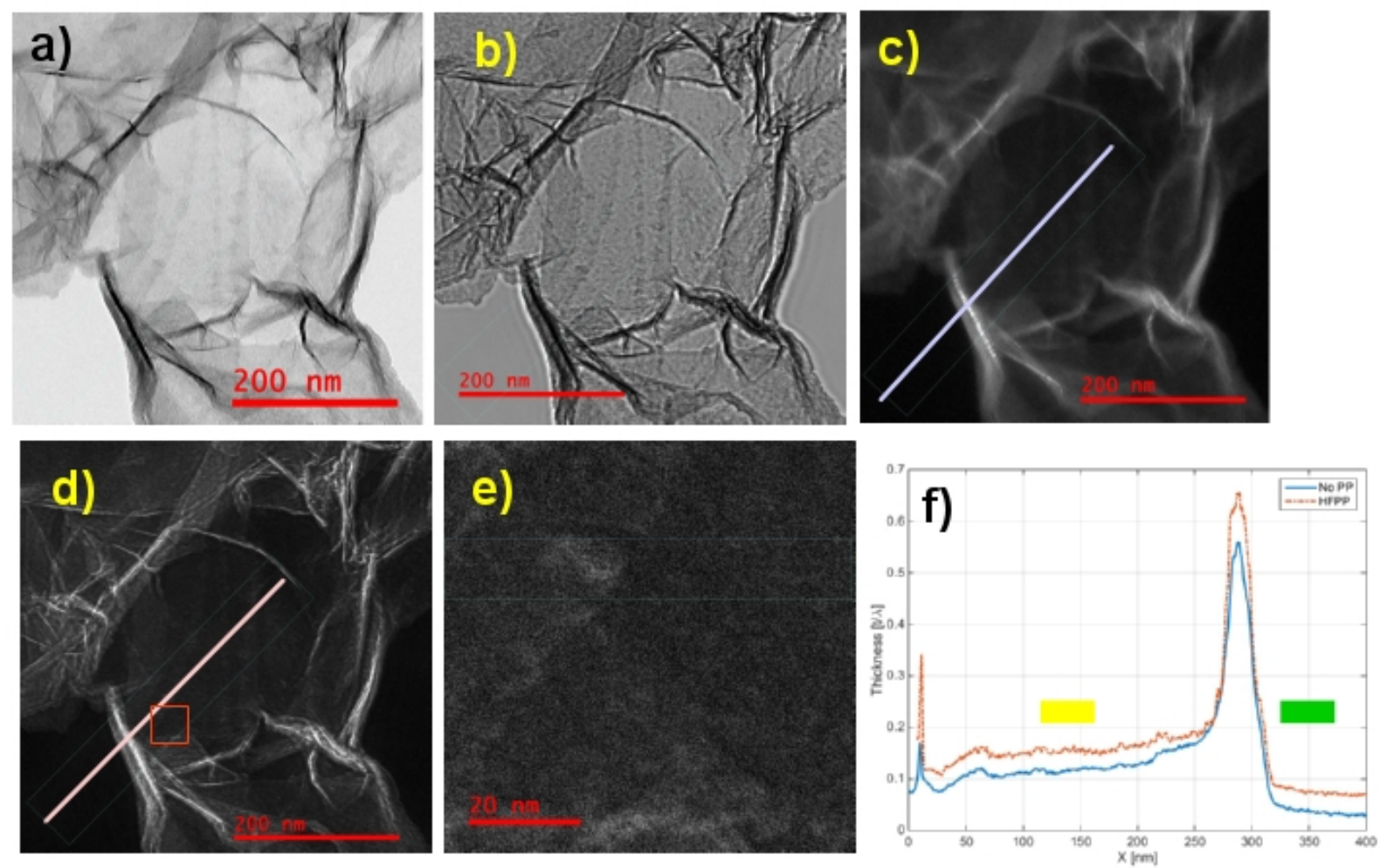

Figure 1 a) Zero-loss filtered BFTEM image b) Zero-loss filtered HFPP image c) standard EFTEM thickness map d) HFPP-EFTEM thickness map. a) to d) were collected at 50kx nominal magnification..e) HFPP-EFTEM thickness map of region marked with red square in d) at $300 \mathrm{kx}$ nominal magnification. f) thickness profiles from c) and d) in units of inelastic mean free path on $y$ axis and position along the profile in $\mathrm{nm}$ on $x$ axis. The region in yellow and green were used to evaluate the thickness difference between the HFPP-EFTEM and standard EFTEM thickness maps in sample and vacuum respectively. The region marked in yellow in $\mathrm{f}$ ) corresponds to a flat region of the sample while the region marked in green corresponds to vacuum in the bottom left corner of c) and d). The scale bar is $200 \mathrm{~nm}$ in a) to d) and $20 \mathrm{~nm}$ in e). 